\title{
Air invasion in a granular layer immersed in a fluid: Morphology and dynamics
}

\author{
Germán Varas · Jean-Christophe Géminard · Valérie Vidal
}

Received: date / Accepted: date

\begin{abstract}
We investigate the morphology and dynamics of the region invaded by air injected at the bottom of an immersed granular bed. Previous experimental results point out the formation of a fluidized zone with a parabolic shape which does not depend, in the stationary regime, on the injection flow-rate. By tilting the experiment, we can tune the effective gravity in the system. We show that it does not affect significantly the morphology either. A numerical study made it possible to access the typical height and width of the structure, which are governed by the relative effects of gravity and capillarity. After a brief review on this subject, we propose first, new experimental observations on the air invasion regimes and on the morphology of the fluidized zone, in particular its growth dynamics; then, we complement the previous numerical study by considering the influence of the bottom boundary condition. In particular, we quantify the morphology of the invaded region when the gas is injected in the bulk, thus when air is likely to propagate downwards. These results are of practical importance in the prediction of the morphol-
\end{abstract}

G.V. acknowledges a grant by CONICYT (Comisión Nacional de Investigación Científica y Tecnológica, Gobierno de Chile) and financial support from FONDECYT Project No.11121300.

\section{G. Varas}

Instituto de Física, Pontificia Universidad Católica de Valparaíso, Avenida Brasil 2950, Valparaíso, Chile.

Previously at Laboratoire de Physique, Université de Lyon, Ecole Normale Supérieure - CNRS, 46 Allée d'Italie, 69364 Lyon Cedex 07, France.

J.-C. Géminard · V. Vidal

Laboratoire de Physique, Université de Lyon, Ecole Normale Supérieure - CNRS, 46 Allée d'Italie, 69364 Lyon Cedex 07, France. E-mail: valerie.vidal@ens-lyon.fr ogy of gas invasion in soils, from $\mathrm{CO}_{2}$ sequestration to pollutant propagation.

Keywords Granular flow · Fluidization · Patterns

PACS 83.80.Fg · 47.57.Gc $\cdot$ 47.85.Dh

\section{Introduction}

Gas invasion in an immersed granular layer is a widespread phenomenon of striking importance in many contexts, from oil industry $[1,2]$ to soil decontamination, where air injection in the subsurface and its subsequent rising by buoyancy removes or chemically degrades the contaminants [3]. In natural processes, piercement structures display many examples of such phenomenon [4]. A highly pressurized fluid (liquid or gas) rises from a given source at depth through sediments or crustal rocks, leading to partial fluidization or liquefaction of the granular matrix. The shape of the fluidized zone, inferred from geophysical measurements, widens upwards, and may form a crater at the surface. These structures are encountered worldwide and at different scales, from kilometers (kimberlite pipes [5], mud volcanoes [6]) to hundreds or tens of meters (hydrothermal vents [7], submarine pockmarcks [8]).

Previous experimental works have been proposed to model this phenomenon in the laboratory. Two-phase flow experiments (gas/grains or liquid/grains) pointed out the existence of three different regimes when the gas or liquid injection flow-rate increases [1,9-11]: at low flow-rates, the system acts as a rigid porous medium and the fluid (gas or liquid) percolates through the granular matrix without any significant grain motion; at intermediate flow rates, the medium starts deforming, with the onset of small-scale instabilities and pip- 
ing; at large flow-rate, explosion at the surface and fluidization of the medium is reported (hydraulic failure).

Many numerical models have been developed, mostly in 2D, to investigate such mechanisms. They include different growth processes such as the Eden cluster model [12], ballistic models [13,14], dendritic growth or the Diffusion-Limited Agregation (DLA) [15]. These models extensively studied the invasion process without external forces (e.g. [16-18]) or, in the last twenty years, the destabilization induced, for example, by the presence of gravity in the system [19-21].

Investigating three-phase flow systems (i.e. the injection of a fluid $\mathrm{A}$ in a granular medium immersed in a fluid B), is even more complex, in particular when it involves the grains displacement and the dynamics of the propagation front. Experiments of air injection in a suspension confined in a horizontal Hele-Shaw cell, where the grain density matches the host fluid, revealed a wide range of morphologies, from the classical SaffmanTaylor instability to fingering in a fixed porous medium [22]. A recent study by Sandnes et al. [23] proposed a unified phase-diagram of the different morphologies of air invasion when injecting air into an immersed loose granular medium confined in a horizontal HeleShaw cell. The introduction of inter-particule friction and sedimentation revealed three pertinent parameters: the granular filling fraction, system stiffness (related to the volume of the air reservoir) and air injection rate. Reports of several invasion morphologies, from stickslip bubbles to fingering or fracturing, were explained in the frame of the transition between a frictional and viscous regime.

In a recent work, we investigated experimentally [24] and numerically [25] the morphology of the region invaded by the gas when air is injected punctually at the bottom of an immersed granular bed in a vertical cell. The influence of the effective gravity in the system can be tuned by tilting the experimental cell or by varying the numerical parameters [25]. From experimental observations we reported two different invasion regimes: percolation and fracturing of the medium. Here, we report a new regime of stable channels, in particular when the effective gravity in the system is low. At long times, a fluidized zone forms and its morphology in stationary state was investigated $[24,25]$. We complement these results by the study of the transient regime and growth dynamics of the fluidized region. Finally, the previous numerical study [25] was inspired by experimental results obtained in a vertical Hele-Shaw cell where the gas was injected locally at the base of the immersed granular material. Thus, in the numerical calculation, we did not permit the gas to propagate downwards below the injection point. In many situations, however,

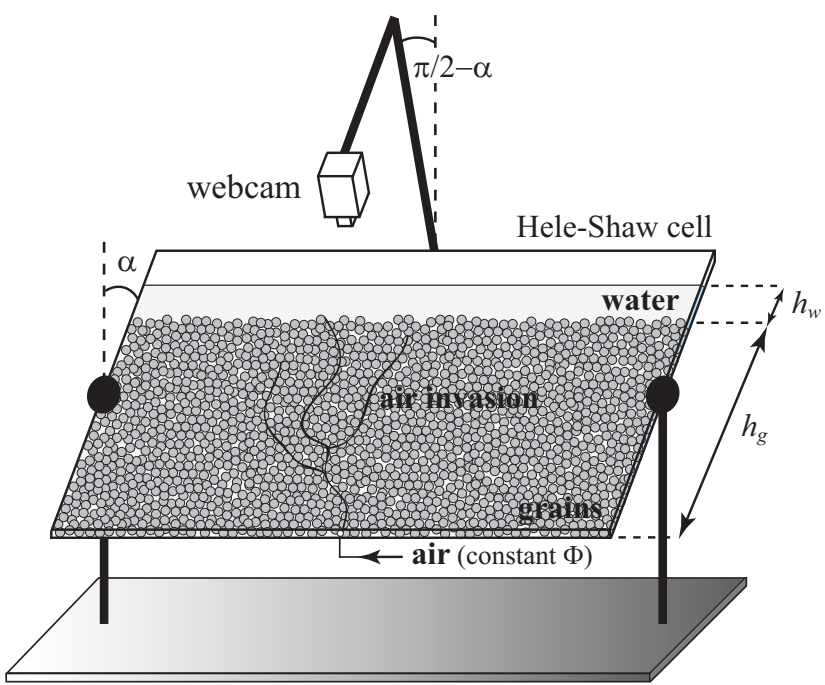

Fig. 1 Experimental setup. Air is injected at constant flowrate $\Phi$ at the bottom of a granular layer immersed in water (see text). The effective gravity in the system is tuned by tilting the cell between $\alpha=0$ (vertical) and $\alpha=\pi / 2$ (horizontal).

the propagation of air in a porous medium, biaised by gravity, is not limited by a solid bottom wall and the gas can explore freely all directions. In particular, we mention the example of liquid injection in the ground for enhanced oil recovery [26], or the recent topic of $\mathrm{CO}_{2}$ storage which aims at reducing greenhouse effect and global warming by injecting carbon dioxide several hundred meters below in the subsurface [27,28]. In this latter case, it is important to estimate the morphology of the region invaded by the gas below the injection point [29]. Any large-scale $\mathrm{CO}_{2}$ release could, indeed, perturbate the carbon-cycle and is likely to induce global climate changes [30]. Here, we complement the previous numerical results [25] by quantifying the morphology of the invaded region when the gas is injected in the bulk, thus when air is likely to propagate downwards. We analyze how the boundary condition at the bottom alters the final morphology of the invasion zone. In particular, we determine the depth reached by the gaseous phase below the injection point.

\section{Experimental observations}

\subsection{Experimental setup}

The experimental setup consists of a Hele-Shaw cell (width $40 \mathrm{~cm}$, height $30 \mathrm{~cm}$, gap $2 \mathrm{~mm}$ ) filled with grains immersed in water (Fig. 1). Air is injected at the bottom of the cell at constant flow-rate $\Phi$ (from 0.17 to $4.3 \mathrm{~mL} / \mathrm{s}$ ) by a mass-flow controller (Bronkhorst, MassStream Series D-5111). The grains are spherical glass 

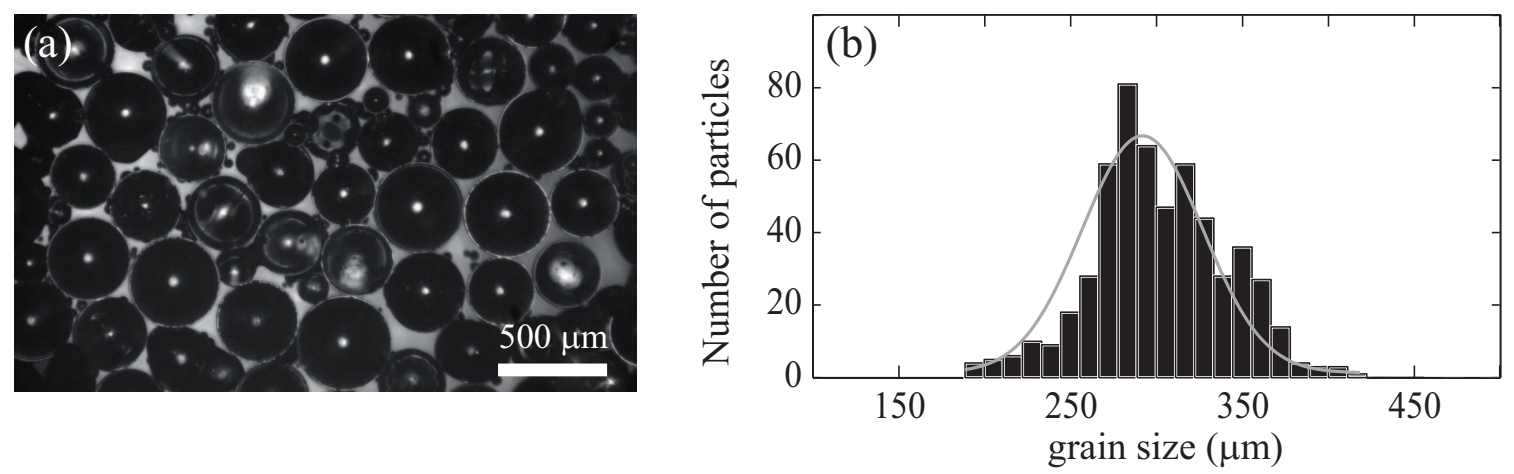

Fig. 2 Particle size distribution of the glass beads used in our experiments. (a) Image of the glass beads. (b) Size distribution for the batch $d=250-425 \mu \mathrm{m}$. The grain size distribution is fitted by a gaussian, giving the average grain size and polydispersity (here, $d=293 \pm 50 \mu \mathrm{m}$ ).

beads (USF Matrasur, sodosilicate glass), previously sieved to control their size. Unless specified, the grains diameter $d$ is in the range $250-425 \mu \mathrm{m}$. The grain average diameter and size distribution are inferred from direct imaging of several samples (about 1000 grains, see Fig. 2). The height of the granular bed is $h_{g}=22 \mathrm{~cm}$, and the water layer above the initial flat free surface of the grains is $h_{w}=2 \mathrm{~cm}$. Images of the air invasion are recorded with a webcam (Logitech, QuickCam S7500, $\left.640 \times 480 \mathrm{px}^{2}\right)$, either in real-time $(20$ images/s) or in time-lapse (1 image every 15 minutes) to quantify the growth of the fluidized zone. The light source consists of a transparency flat viewer (Just NormLicht, Classic Line) positioned behind the cell. The effective gravity in the system can be tuned by tilting the cell with an angle $\alpha, g_{\text {eff }}=g \cos \alpha$, going from vertical ( $\alpha=0$, $g_{\text {eff }}=g$, the acceleration due to Earth gravity) to horizontal $\left(\alpha=\pi / 2, g_{\text {eff }}=0\right)$. For sake of clarity, the angle $\alpha$ is given in degrees in the following, from $\alpha=0^{\circ}$ (vertical cell) to $\alpha=90^{\circ}$ (horizontal cell).

The initial condition is prepared as follows. First, we pour the grains up to the height $h_{g}$. We then fill the entire cell with water, up to the top boundary, and close the upper aperture. Hence we flip the cell bottom-up, which forces most of the small bubbles which may be trapped in the system to separate from the surrounding grains and rise to the surface. While the grains are not fully deposited (very loose packing) and still sediment, we flip the cell back to its original position. All the small bubbles initially trapped in the bulk are therefore extracted from the system. We let the grains deposit gently, then fix precisely the water, $h_{w}$, and grain height, $h_{g}$. This latter is gently levelled with a small paddle. This prococol ensures a reproducible initial condition (loose packing). Note however that the global packing is controlled but not the local packing, which will lead to different observed invasion patterns (see section 2.2).

\subsection{Air invasion at short times}

The air invasion at short times displays different patterns depending on the injected flow-rate $\Phi$ and on the tilt angle $\alpha$. Figure 3 presents a tentative phase diagram in the $\Phi-\alpha$ plane. For high effective gravity (low tilt angle), two patterns are reported. At low flow-rate, the air invades the system without significantly moving the grains (percolation). We observe several branches in the medium and, consequently, simultaneous gas emission loci at the surface. At high flow-rate, the air fractures the system and propagates in the bulk by pushing the grains aside. We still observe branches, but much less numerous than in the percolation process. In this case, the upper branch always reaches first the layer surface, and only a single gas emission point can be observed at a time. After the gas emission, the pressure drops in the fracture and this latter closes. The pressure at the injection point subsequently increases again, until a new fracture is opened in the system.

The transition between the percolation and fracture regime is not sharp. Over a wide range of intermediate flow-rate, it is impossible to predict the air invasion pattern in the system. Indeed, the dynamics depends not only on the flow-rate, but also on the local heterogeneities (due to polydispersity and compaction), which are not known and not reproducible from one experiment to another.

We report the existence of a threshold angle $\left(\alpha_{c} \simeq\right.$ $\left.60^{\circ}\right)$ above which the air opens a channel through the granular medium, and the effective gravity is not able to close it anymore. We then observe the formation of channels (Figs. 3c,d) which remain stable over long 


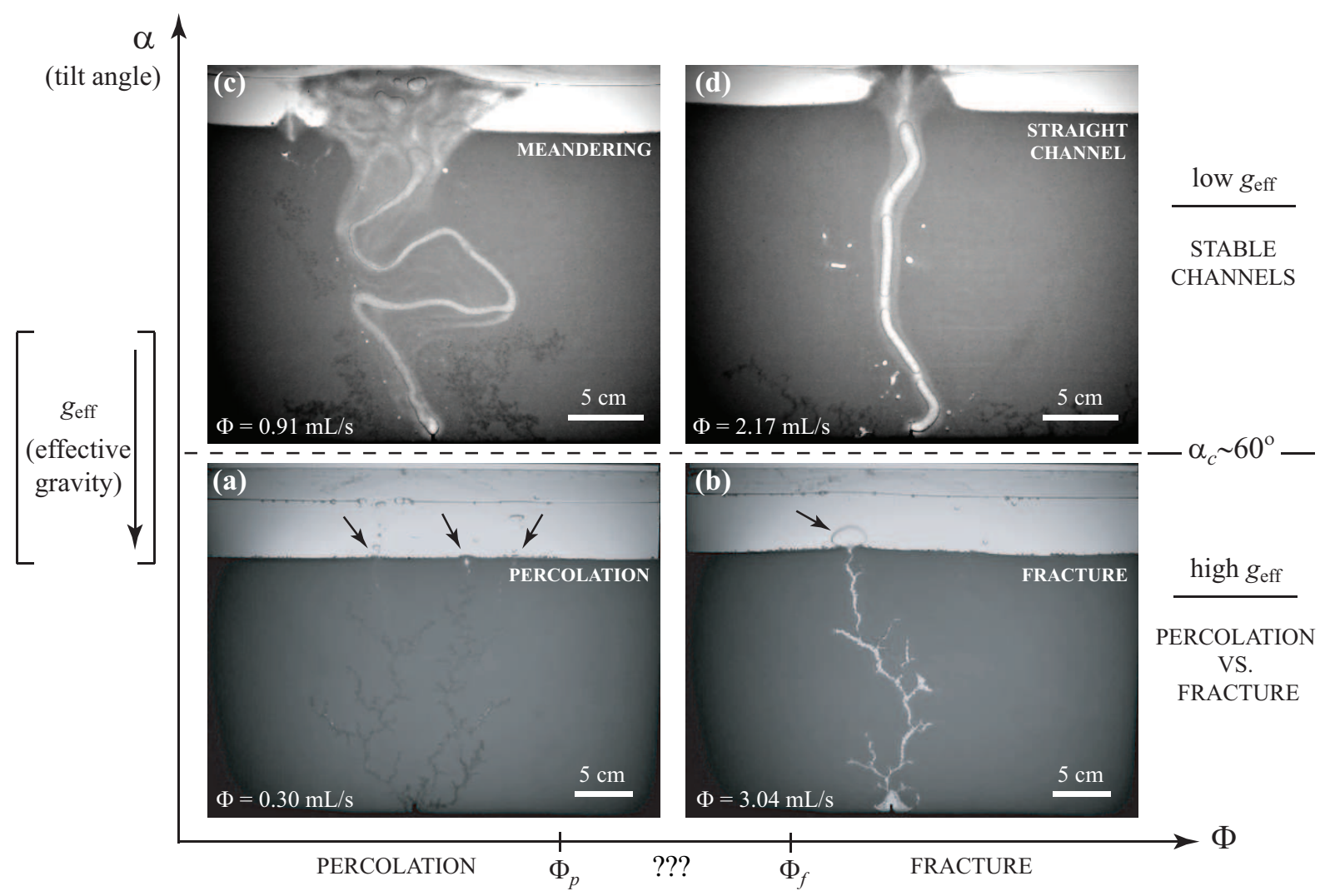

Fig. 3 Tentative phase diagram in the $\Phi-\alpha\left(\Phi-g_{\text {eff }}\right)$ plane. (a,b) For high effective gravity $\left(\alpha<60^{\circ}, g_{\text {eff }}>g / 2\right)$, the air invades the granular layer at short times with different dynamics. (a) At low flow-rate $\left(\Phi<\Phi_{p}\right)$, we always observe percolation. (b) at high flow-rate $\left(\Phi>\Phi_{f}\right)$, the air systematically fractures the system. Note that for intermediate flow-rates $\left(\Phi_{p}<\Phi<\Phi_{f}\right)$, it is impossible to predict the initial air invasion morphology (percolation or fracture, see text). Black arrows indicate the gas emission at the surface, at simultaneous locii for percolation while at a single point for fracture. (c,d) For low effective gravity $\left(\alpha \geq 60^{\circ}, g_{\text {eff }}<g / 2\right)$, we observe the formation of stable channels. These structures display different morphologies depending on the injection flow-rate, from (c) meandering at low flow-rate to (d) straight channels at high flow rates. At long times, the channels eject the grains from the initial layer and it is not possible to define a fluidized zone anymore.

times (typically, hours). These channels display different geometries, from tortuous channels at low flow-rate (Fig. 3c) to straight channels at high flow-rate (Fig. 3d). It is interesting to note that these latter patterns were not reported in [23], maybe due to the fact that the flowrates used in our experiments are much higher than the ones used by these authors. At long times, in the channelling regime the air ejects the grains out of the initial layer, and the central part of the cell contains no grains anymore - only air rising through a water layer. Therefore, this regime has not been studied further, and the following sections only concern the regimes for which $\alpha<\alpha_{c}$.

\subsection{Steady state: morphology of the fluidized zone}

The repetitive air rises through the system lead to the formation of a fluidized zone. After a time long enough - typically, from one hour up to 20 hours depending on the imposed flow rate - the fluidized zone reaches a stationary shape. It has been shown in a previous work [24] that, for a vertical Hele-Shaw cell, this shape is parabolic and independent of the injected flow-rate, i.e. of the initial invasion pattern (percolation or fracture).

To quantify the morphology of the fluidized zone as a function of the injected flow-rate $\Phi$ and the angle $\alpha$, we considered, for each set of parameters, the steady state for which the contour of the fluidized region does not evolve significantly anymore. For each angle, the total duration of an experiment (at given $\Phi$ ) is fixed so that the total volume of air injected in the system is the same. The experiment can thus last from several hours to tens of hours, depending on the injection rate. One image every 15 minutes is recorded.

The contour of the fluidized zone is determined as follows. For a given experiment, we compute the difference between successive images, then stack the resulting sequence (Fig. 4a). The contrast obtained by this 

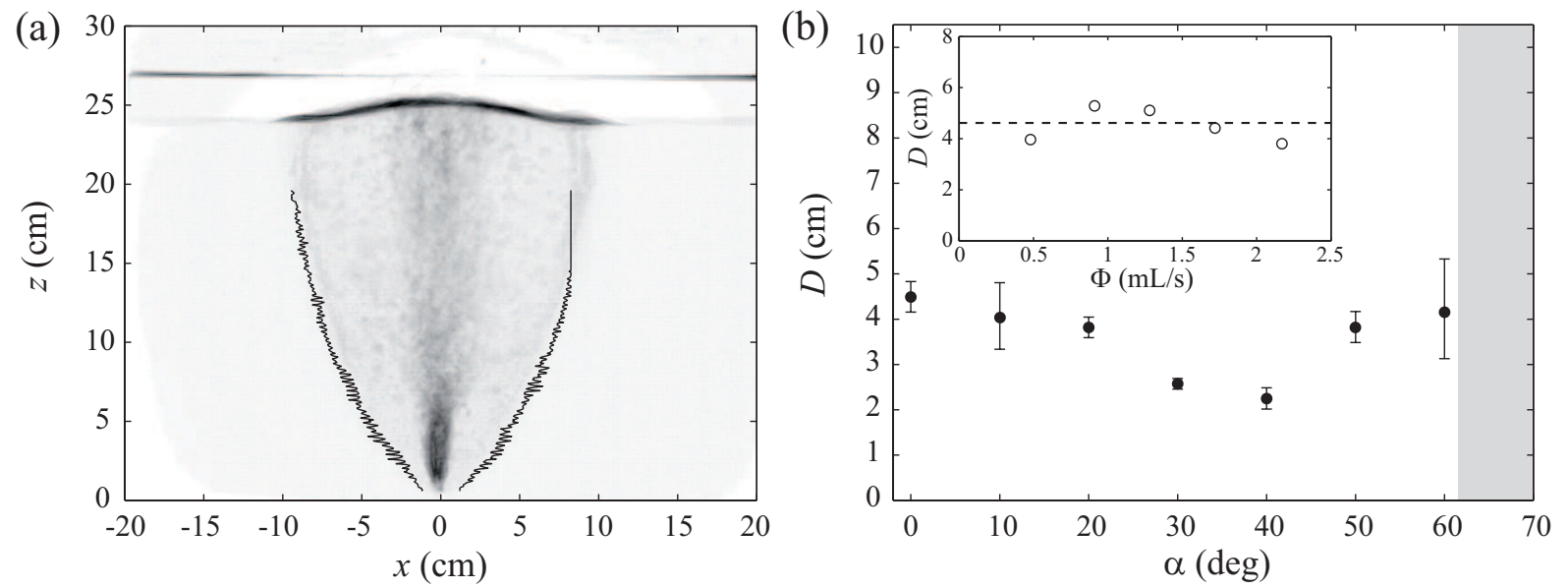

Fig. 4 (a) Example of the final invasion image obtained by superimposing the images sequence $[\Phi=0.91 \mathrm{~mL} / \mathrm{s}]$. In white are regions where the grains did not move in the layer, while darker gray indicates regions where the grains moved a lot. The contour of the fluidized zone (black lines) is obtained by automatic detection. The detection is limited to $z \leq 16 \mathrm{~cm}$ to avoid the deformations due to the free surface. (b) Diffusion coefficient $D$ as a function of the tilt angle $\alpha$. The points and error bars for a given angle are obtained from the mean and standard deviation of $D$ vs. $\Phi$ (e.g. $D=4.5 \pm 0.7 \mathrm{~cm}$ for $\alpha=0^{\circ}$, inset). For $\alpha>60^{\circ}$ (gray zone), it is not possible to define a fluidized zone anymore (see text).

(a)

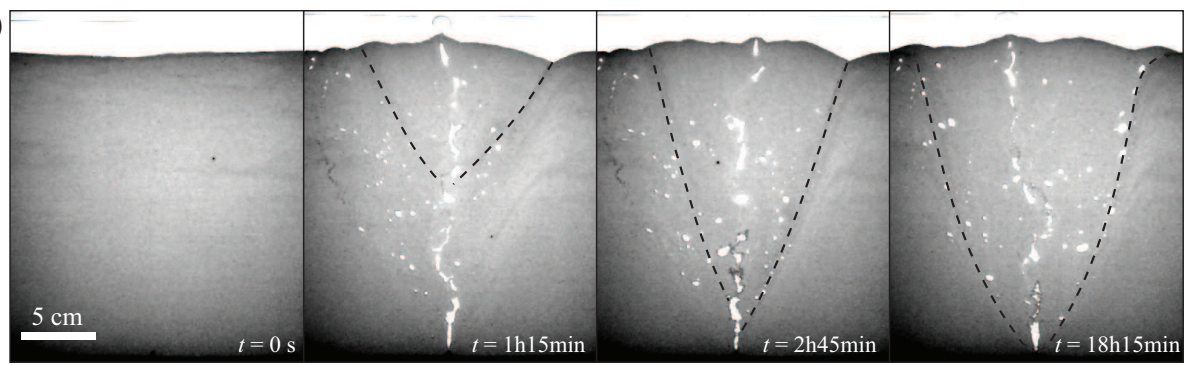

(b)

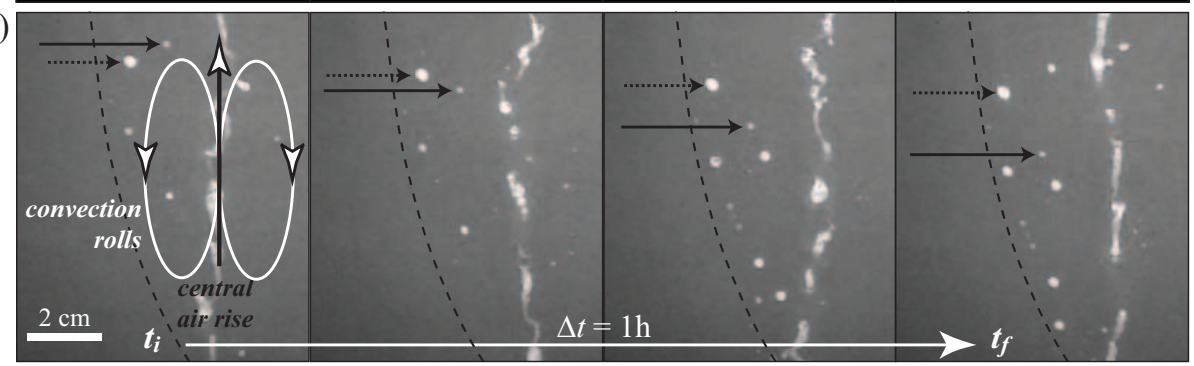

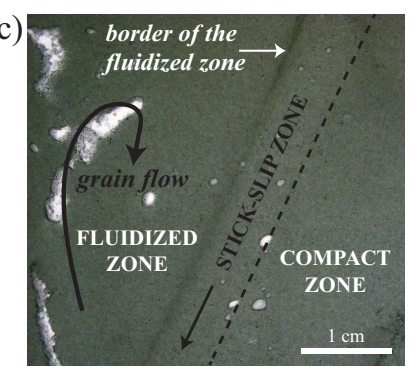

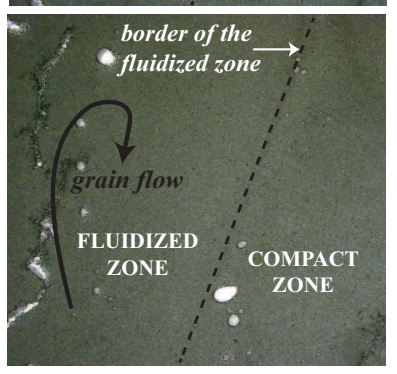

Fig. 5 (a) The growth of the fluidized zone (dashed lines) starts from the granular bed surface and propagates downwards. (b) Zoom on the fluidized zone (for clarity, its left contour only is represented by the dashed line). The air rising through the grain layer (central arrow) generates two convection rolls (in white). The downward flow velocity decreases close to the fluid zone outer border. The horizontal black arrows follow the position of two bubbles trapped in the fluidized zone for about 1 hour $\left[\Phi=0.91 \mathrm{~mL} / \mathrm{s}, \alpha=0^{\circ}\right]$. (c) Top: The stick-slip zone (SSZ), located between the core of the fluidized zone and the outer compact zone, is characterized by discrete events (see text). Bottom: This zone disappears in stationary regime, when the fluidized zone reaches its final geometry $[\Phi=2.17 \mathrm{~mL} / \mathrm{s}]$.

method is good enough to detect automatically the contour of the fluidized region (black line, Fig. 4a). This contour indicates the region at the center where the grains have moved. To avoid any effect of the free surface, we limit the detection until about $2-3 \mathrm{~cm}$ below the grain surface. As already shown in previous work [24], the fluidized zone exhibits a parabolic shape, $x=\sqrt{D z}$, and can be interpreted in terms of a diffusive process, where $D$ (here, a length) is the analog of a diffusion coefficient, by considering that the vertical axis $z$ plays a role analog of time in a classical diffusion process.

No significant variation of $D$ as a function of $\Phi$ is reported, despite the very different patterns of air invasion at short times for small (percolation) and large (fracture) $\Phi$ (Fig. 4b, inset). For each $\alpha$, we report in Fig. $4 \mathrm{~b}$ the mean and standard deviation obtained from 
(a)

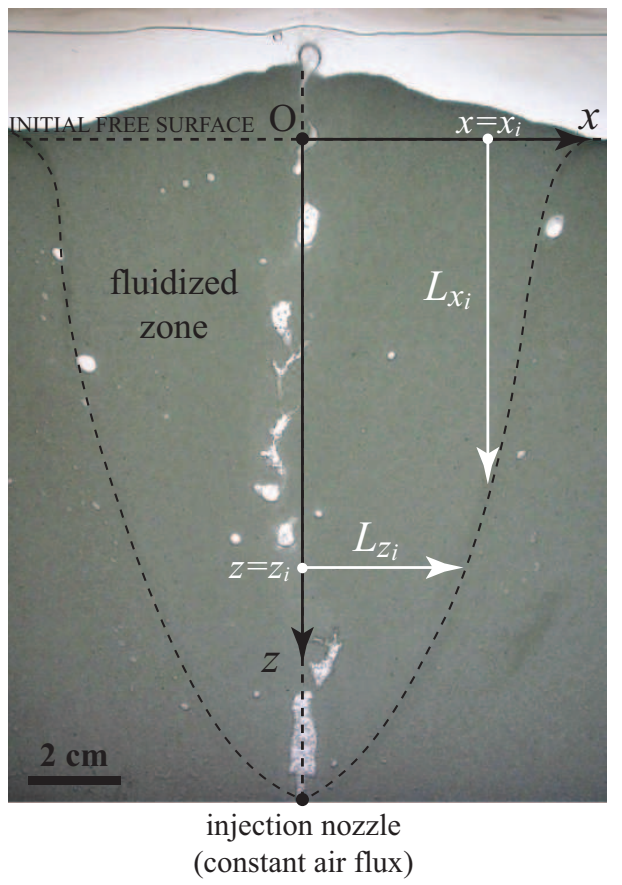

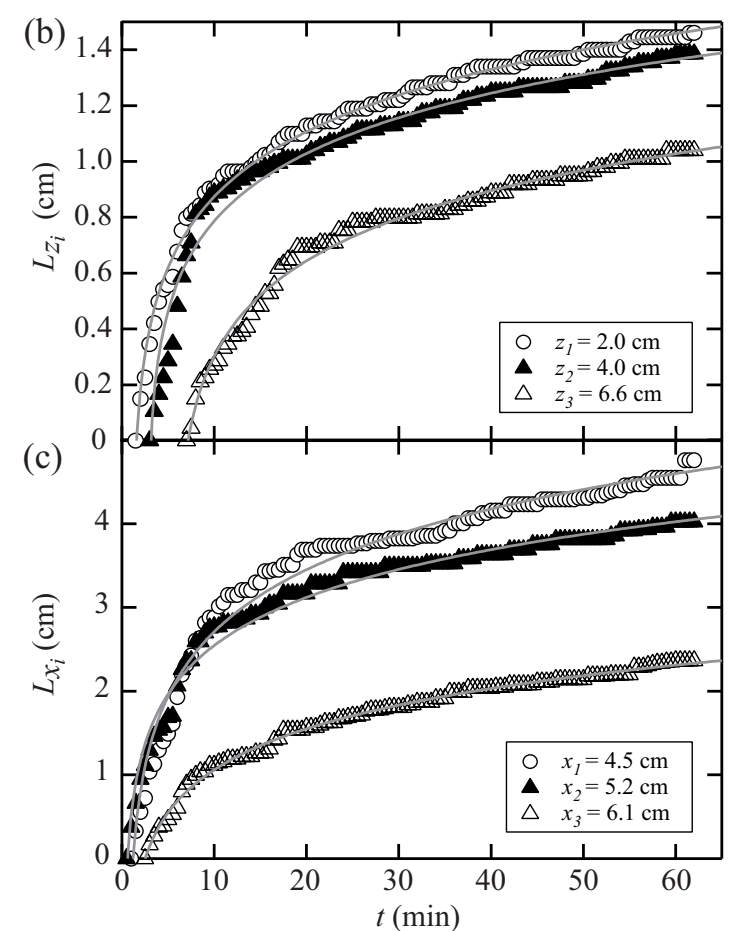

Fig. 6 Temporal evolution of the contour of the fluidized zone in the transient state. (a) Example of a fluidized zone in the transient state. Its contour (dashed line) has not reached yet its final position. We consider the evolution of this contour along the horizontal $\left[L_{z_{i}}\right]$, for a fixed vertical position $z_{i}$, and along the vertical $\left[L_{x_{i}}\right]$, for a fixed $x_{i}$. The origin of $(x-z)$ axis is taken at point $O$. (b) Temporal evolution of $L_{z_{i}}$ for different vertical positions $\left(z_{1}, z_{2}, z_{3}\right)$ below the free surface; (c) Temporal evolution of $L_{x_{i}}$ for different horizontal positions $\left(x_{1}, x_{2}, x_{3}\right)$ [gray lines: logarithmic fit].

measurements at different injected flow-rates. By tilting the experimental cell, i.e. decreasing the effective gravity in the system, we observe a slight decrease of $D$, which then increases back to a roughly constant value $D \sim 4 \mathrm{~cm}$. This non-trivial evolution cannot be explained by a simple analytical [24] or numerical [25] model based on a rigid grain matrix and considering the different paths explored by the air in the system. Indeed these simple models, which provide an interpretation to the parabolic shape of the fluidized zone, predict a continuous increase of $D$ as a function of $\alpha$ (see [24], Fig. 7 or [25], Fig. 8). The grains displacement and dynamics, which are not taken into account in these simplified models, play a fundamental role in the fluidized zone formation.

\subsection{Transient regime: growth dynamics}

We observe that the fluidized zone starts forming from the granular bed surface, where the grains are not constrained and can rearrange easily. It then progressively widens and grows downwards (Fig. 5a). This downward growth of the fluidized region has been recently pointed out in similar experiments by Kong et al. [31]. Interestingly, this geometry and evolution are reminiscent of the draw bodies observed in the mining industry, which are formed by drawing out fragments from an extraction point at the base of the ore bed [32]. Note that the borders of the fluidized zone are well-defined due to the density contrast between the central fluidized zone (loose packing) and the compact outer zone. The bubbles which get trapped during the experiment in the fluidized zone act as tracers and can be followed in time (Fig. 5b). We thus report that, on both sides of the central air rise, two convection rolls are generated, which entrain the grains upwards close to the center, and downwards close to the fluidized zone border (Fig. 5b, arrows).

To quantify the growth dynamics of the fluidized zone, we report in Figure 6 the temporal evolution of the distance $L_{z_{i}}$ [resp. $L_{x_{i}}$ ] between the central axis of air rising (resp. the free surface) and the outer border of the fluidized zone at time $t$, for a given height $z_{i}$ (resp. a given distance $x_{i}$ from the central axis, see Fig. 6a). The horizontal position of the front, $L_{z_{i}}$, increases rapidly at short times $(t<10 \mathrm{~min})$ then slows down (Fig. 6b). The data can be fitted by a logarithmic law (gray lines, Fig. 6b). The front propagation along the vertical, $L_{x_{i}}$, is quicker than along the horizontal (Fig. 6c, compare 
the scale on the $y$-axis) and can also be fitted by a logarithm (gray lines, Fig. 6c).

Finally, it is interesting to note that, in the transient regime, we observe a well-defined region between the fully fluidized zone (grains always in motion) and the compact outer zone. In this transition zone, phenomena similar to the well-known stick-slip mechanism occur: the grains move downwards by successive slides (black arrow, Fig. 5c, top). This dynamics can be interpreted as a consequence of the shear induced on the fluidized zone border by the downward grain-flow associated with the convection roll in this region. The stick-slip zone (SSZ) evolves in time: its width decreases while the fluidized zone increases, until it disappears completely in the steady regime (Fig. 5c, bottom).

\subsection{Conclusion on the experimental part}

This section summarized the experimental results, up to date, on the morphology and dynamics of air invasion in an immersed granular bed. In the presented configuration, the air invasion is limited by the solid bottom boundary conditions, and the gas cannot propagate downwards.

In the following section, we complement the numerical results we obtained in our previous works by introducing the case of a free bottom boundary condition. In particular, we compare the morphology (width, height, shape) of the invasion zone. These results concern the case of a rigid grain matrix, with air percolating through the system.

\section{Numerical model}

\subsection{Description}

The procedure to determine the morphology of the invasion zone is the same as described in [25]. In a simple approach, we consider that the gas is opening a path in a square matrix of fixed grains. In the above experiment, the fluidized zone is created by the successive air bubbles rising through the system. In the numerical model, by analogy, this zone corresponds to the region explored by the successive air paths through the system. The final morphology of both processes is comparable, in the sense that the multiple events of air rising through the system generate either the fluidized zone (experiments) or the region explored by the air (numerical model). Although this modeling does not take into account the grains motion in the so-called fluidized zone, it points out the relevant parameters to describe its morphology, as already shown in previous works [24, $25]$.

In the model, each saddle point between two vertices (void space between the grains) is associated with a different threshold in the capillary overpressure that the gas must overcome to cross from a given vertex to an adjacent one. The threshold distribution thus accounts for the heterogeneity of the system at the grain scale. The typical overpressure is of the order of $\Delta P \sim \gamma / r$ where $\gamma$ stands for the gas-liquid surface energy and $r=d / 2$ for the typical radius of the grains. We denote $\sigma_{P}$ the width of the distribution of the capillary overpressures in the system.

While air is injected, a new vertex is invaded by the gas when, anywhere along the path, the difference between the (homogeneous) pressure in the gas and the local pressure in the surrounding liquid overcomes the local threshold in the capillary overpressure. Considering that, due to hydrostatics, the pressure in the liquid depends on the depth, one understands that the path grows mainly in a region of typical height $h \sim \sigma_{P} / \rho g$ at its top. Note that the growth mechanism allows the formation of the side-branches as long as $h$ is larger than the typical size of the grains $r=d / 2$. In this context, the parameter

$\chi \equiv \frac{2 \sigma_{P}}{\rho g d}$,

such as $h=\chi r$, appears as a natural dimensionless control parameter of the system (see [24] for the full mathematical introduction of the parameter $\chi$ ). It can be rewritten

$\chi \sim \frac{2 w \gamma}{\rho g d^{2}}$

by denoting $w$ the typical variation of the pore size (typically, a few \%) and assuming that their size is $\sim d$. Note that this parameter slighly differs from the classical Bond number used to characterize the flow regimes of fluid invasion in saturated porous media under the influence of gravity, $B_{0}=\Delta \rho g \delta^{2} / \gamma$, where $\Delta \rho$ is the difference in density between the resident and invading fluids, and $\delta$ the characteristic pore size. The Bond number, indeed, does not account for the grain (or pore) polydispersity, which is included in the parameter $\chi$.

For large values of $\chi$ the invaded region widens, thanks to the formation of side-branches, whereas small values of $\chi$ are associated with poorly branched, almost vertical paths. While the simplistic model proposed in [24], neglecting the formation of side branches, can be solved analytically, accounting for the formation of side branches brings forward the need for a numerical modelling.

To get statistical information on the geometry of the invaded region, we create a large number (2000) of nu- 
(a)

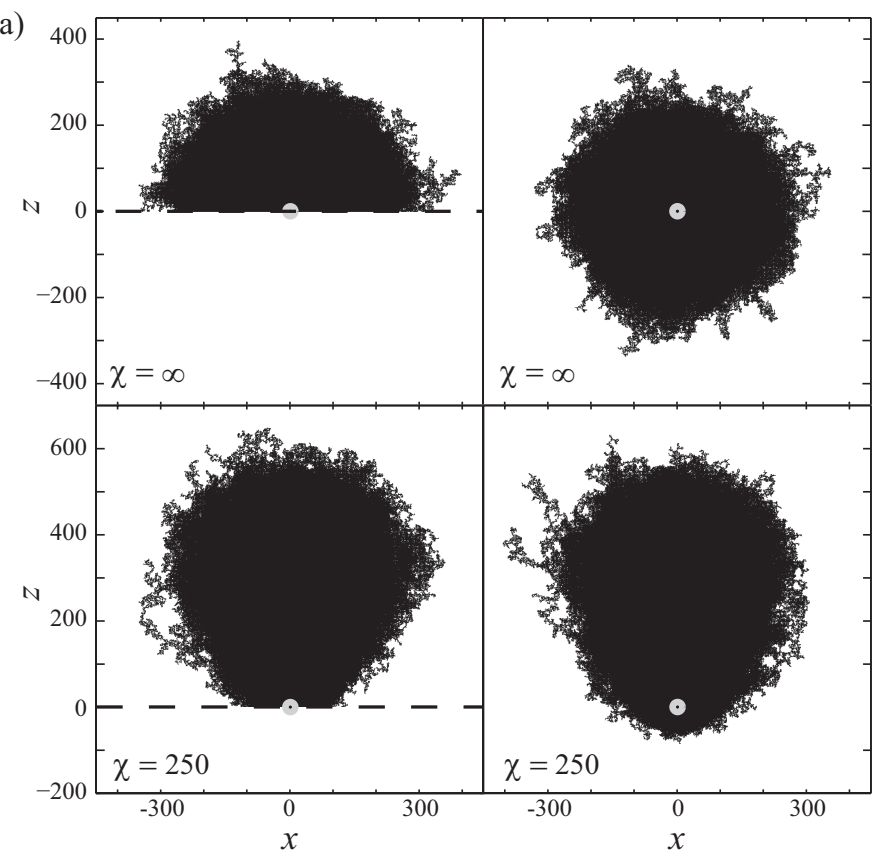

(b)

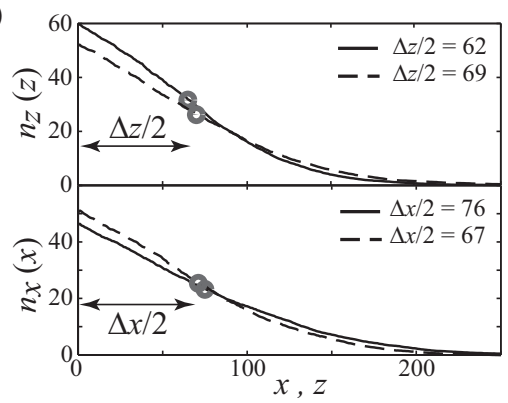

(c)

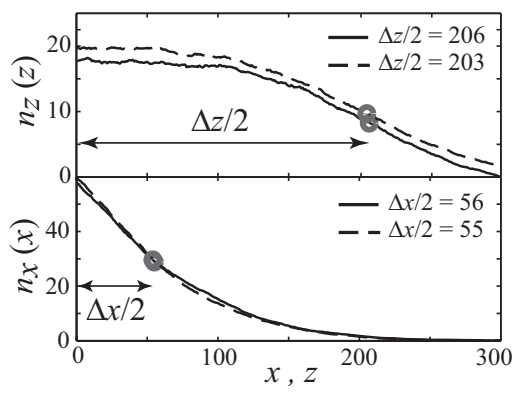

Fig. 7 (a) Numerical results obtained by superposition of 2000 air paths through the system with (left) and without (right) bottom wall [gray dot $=$ injection point]. The global geometry is not significantly altered by the bottom boundary condition. $(\mathrm{b}, \mathrm{c})$ Global intensity profiles along the $x$-axis, $n_{x}(x)$, and $z$-axis, $n_{z}(z)$ from the images reported in (a) [solid lines, with bottom wall; dashed lines, without bottom wall]. The dots indicate the value at half height, taken as the typical size of the invasion zone along the $x$-axis $(\Delta x)$ and the $z$-axis $(\Delta z)$ (see text). For (b) $\chi=\infty$ (no gravity) the difference in $\Delta x$ and $\Delta z$ between the two boundary conditions is of about $10 \%$ whereas for (c) $\chi=250$ the difference is of the order of $2 \%$.

merical paths for given values of the control parameters. To do so, the pressure thresholds are chosen according to a Gaussian distribution of width $\sigma_{P}$, the value of $\chi$ is fixed and a number $N$ of individual steps is considered. As $N$ stands for the number of voids between the grains invaded by the gas, $N$ accounts for the amount of gas injected in the system. Indeed, denoting $v_{\mathrm{p}}$ the typical volume associated with the void space between the grains, the total gaseous volume in the system is $V \equiv N v_{\mathrm{p}}$.

\subsection{Influence of the bottom boundary condition}

First, we compare the morphologies of the invaded regions when air is injected at the bottom edge of a granular bed (the gas cannot propagate below the injection point) or in the bulk (the gas can propagate downwards from the injection point). Typical results are reported in Fig. 7. At first sight, the boundary condition does not much change the morphology of the invaded region. However, as already reported in [25], the presence of a rigid bottom wall makes impossible, for instance, a perfectly isotropic invasion of the medium when gravity effects are suppressed $(\chi=\infty$, Fig. 7a, top left) whereas the expected isotropy is recovered when the gas is injected in the bulk (Fig. 7a, top right). A quantitative characterization of the geometry is obtained by measuring the typical width $\Delta x$ and height $\Delta z$ of the invaded region. To do so, from the superposition of the 2000 paths (Fig. 7a), we determine the global intensity profile along the $x$-axis, $n_{x}(x)$ (Fig. 7b, bottom), by stacking all the horizontal profiles over the whole height of the system. We consider the typical size $\Delta x$ of the invasion pattern along the $x$-axis to be the width at half the peak value [25]. In the same way, we consider the global intensity profile along the $z$-axis, $n_{z}(z)$ (Fig. 7b, top), by stacking all the vertical profiles over the whole width of the system. We observe that from the inlet, the profile is almost flat and suddenly decreases above a given altitude. In order to compare the two boundary conditions, we define $\Delta z$, the typical size of the invasion pattern along the $z$-axis, as the altitude, above the injection inlet, of the point corresponding to half the plateau value. The region below the inlet will be discussed later.

The comparison of $\Delta z$ and $\Delta x$ for both boundary conditions reveals a difference of about $10 \%$ for $\chi=\infty$ (no gravity) and of about $2 \%$ for $\chi=250$ (Fig. $7 \mathrm{~b}$ and c). As expected, for large $\chi$ (e.g. small gravity effects) the bottom wall plays a significant role by restricting the air path to the upwards direction. For smaller values 

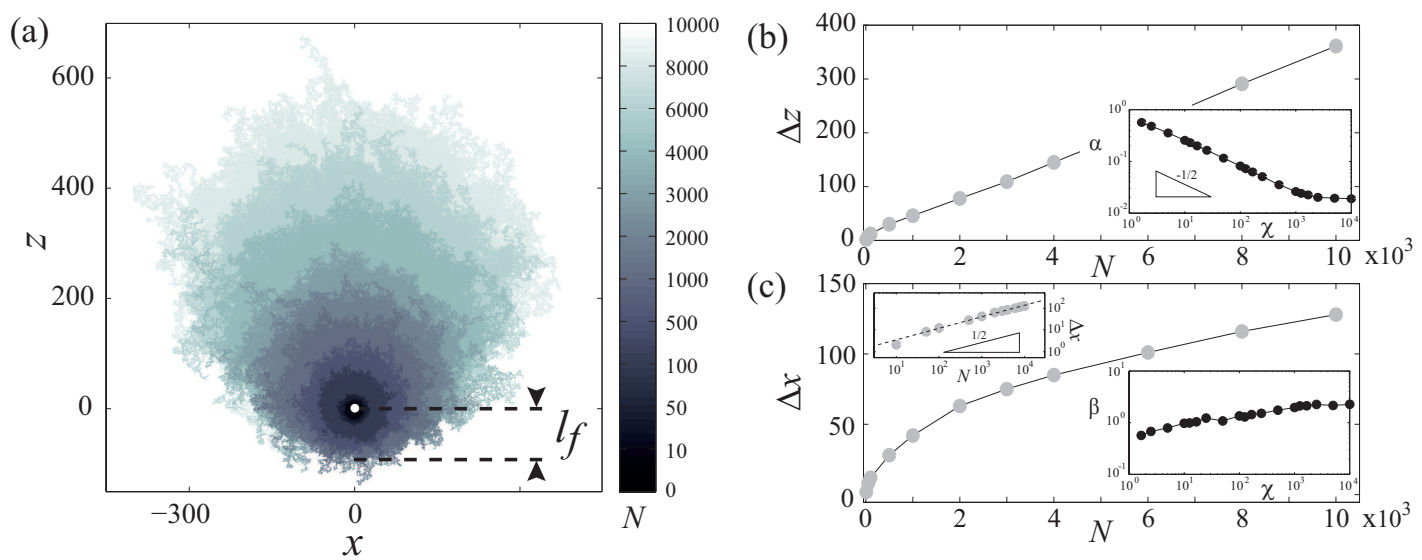

Fig. 9 (a) Shape of the invaded region for different volumes $N$ [superposition of 2000 paths for each value of $N, \chi=500$ ]. The white dot indicates the injection point, and $l_{f}$ denotes the distance over which the air invades the medium downwards (see text). Note that the invasion for small volumes is almost circular, while it elongates in the vertical direction when $N$ increases. (b) The typical height $\Delta z$ of the invaded region is a linear function of the injected volume $N, \Delta z=\alpha N$. Inset: Coefficient $\alpha$ as a function of $\chi$. (c) The typical width $\Delta x$ of the invaded region grows as the square root of the injected volume $N, \Delta x=\sqrt{\beta N}$ (inset, up left). (Inset, down right) Coefficient $\beta$ as a function of $\chi$.

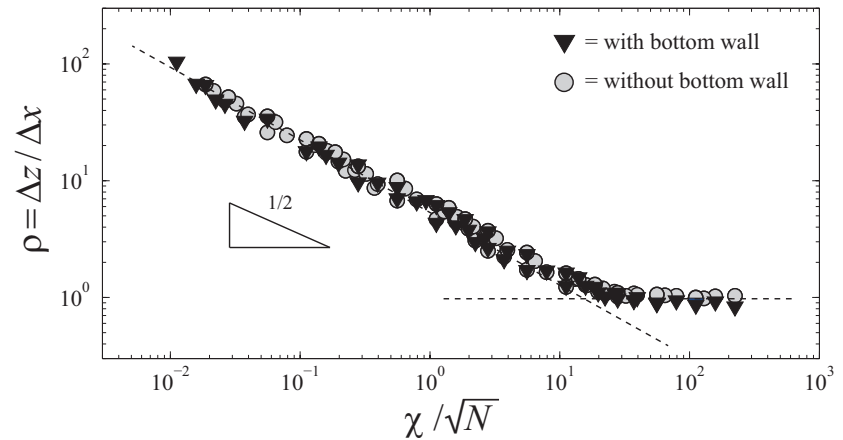

Fig. 8 Aspect ratio $\rho=\Delta z / \Delta x$ of the invasion pattern vs. $\chi / \sqrt{N}$ for the two types of bottom boundary conditions, with (black triangles) and without (gray circles) bottom wall. We observe a good collapse of the data in the whole range of parameters. For $\chi / \sqrt{N} \leq 10$, we find $\rho \propto 1 / \sqrt{\chi}$.

of $\chi$ (e.g. larger gravity effects), the structure is more elongated along the vertical, and the role played by the bottom boundary condition is minimized. It is interesting to note that even in the case where the bottom wall apparently plays a significant role, the typical size of the invasion pattern in both the horizontal and vertical directions, as defined by our statistical analysis, is not significantly altered.

Figure 8 displays the aspect ratio $\varrho=\Delta z / \Delta x$ of the invasion pattern as a function of $\chi / \sqrt{N}$ for the two bottom boundary conditions. We observe that both sets of data collapse over almost all the range of $\chi / \sqrt{N}$ (We remind here that the typical size of the structure scales like $\sqrt{N}$ ). The bottom boundary condition therefore does not alter significantly the global geometry of the invasion zone. We find the following scaling law, common to both conditions for $\chi / \sqrt{N} \leq 10$ :

$\varrho \propto 1 / \sqrt{\chi}$.

For larger $\chi / \sqrt{N}, \varrho$ tends toward an asymptotic value which slightly depends on the bottom boundary conditions $(\varrho=1$ or 0.8 for a free or solid bottom boundary, respectively).

\subsection{Growth dynamics}

To quantify the growth dynamics of the structure, we display the morphology of the invasion pattern as a function of $N$, i.e. of the injected volume (Fig. 9). Results obtained with the bottom wall can be found in [25], we only report here the results associated with the injection in an infinite medium (without bottom wall). The typical height of the invasion zone varies linearly with the injected volume, $\Delta z=\alpha N$ (Fig. 9b), while its width scales as the square root of the volume, $\Delta x=\sqrt{\beta N}$ (Fig. 9c). The variation of coefficients $\alpha$ and $\beta$ as a function of the dimensionless parameter $\chi$ is shown in Fig. $9 \mathrm{~b}$ and $9 \mathrm{c}$, respectively. For $\chi<10^{3}$, we observe that $\alpha \propto \chi^{-1 / 2}$, while $\beta$ only displays a slow increase over the whole range of $\chi$. Note that for smaller gravity effects in the system $\left(\chi>10^{3}\right)$, the invasion pattern is almost isotropic and $\Delta z$ is no longer linear in the injected volume. The isotropy being recovered, $\Delta z$ exhibits the same diffusive growth as $\Delta x$ and increases like $\sqrt{N}$. 

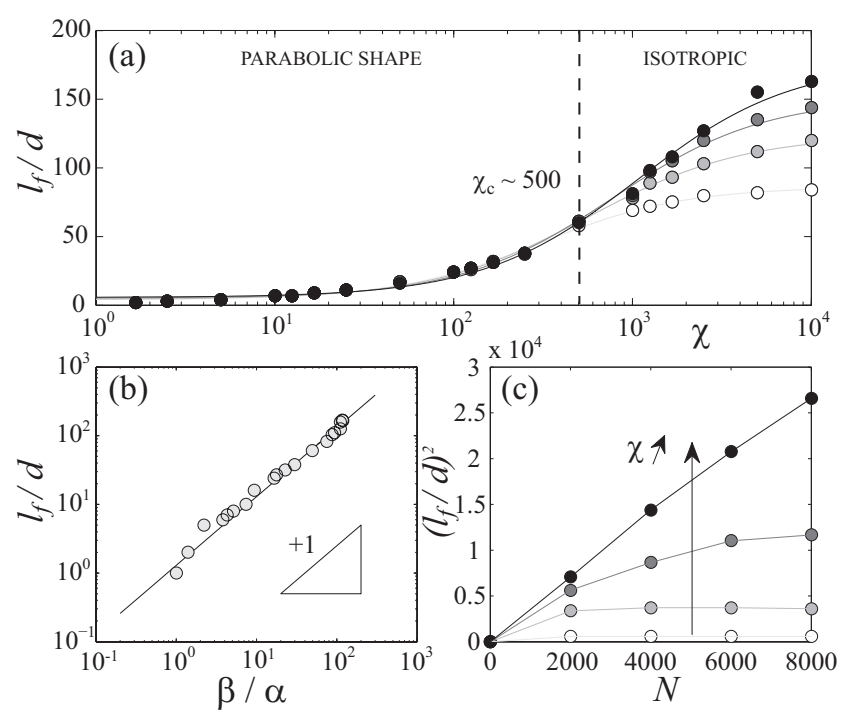

Fig. 10 (a) Distance $l_{f}$ of downward gas penetration, normalized to the grain size $d$, as a function of $\chi$. Fitting curves, sigmoids, are given as eye leads. The results show a transition at $\chi_{c} \simeq 500$. For $\chi<\chi_{c}$, the volume does not play a role in the downward invasion while for $\chi>\chi_{c}, l_{f} / d$ increases when $N$ increases [for white, light gray, dark gray and black symbols, $N=2000,4000,6000$ and 8000 , respectively]. (b) For $\chi \ll \chi_{c}, l_{f} / d$ depends linearly on $\beta / \alpha$ as $l_{f} / d=1.3 \beta / \alpha$ (solid gray line). (c) $\left(l_{f} / d\right)^{2}$ as a function of $N$. Lines are guides for the eye $\left[\chi=100,500,1.67 \times 10^{3}, 10^{4}\right]$.

\subsection{Downward extent of air invasion}

Finally, we focus on the morphology of the invasion pattern in the vicinity of the injection point. In practical situations, when injecting gas inside a granular matrix (e.g., soils), it is crucial to know the depth reached by the gas below the inlet. To do so, we determine from the invasion pattern the invasion contour. This latter is computed by considering arbitrarily a threshold of the occupation fraction, here $f=1 \%$. In other words, the contour corresponds to vertices in the matrix which are occupied by $2000 f$ points, resulting from the superposition of the 2000 paths in the system. We then extract the distance $l_{f}$ between the injection point and the bottommost point of the invasion contour (Fig. 9a).

For a given injected volume, $l_{f} / d$ increases as a function of $\chi$ (Fig. 10a) with an inflexion point at about $\chi_{c}=500$. This transition point, of the same order of magnitude as the one found for the parameter $\alpha$ (Fig. 9), corresponds to the transition between a roughly parabolic shape and a more circular invasion pattern. Note that for large $\chi, l_{f} / d$ tends towards a plateau value, corresponding to the distance reached by an isotropic invasion of the gas in the system. For $\chi \ll \chi_{c}$, $l_{f} / d$ does not depend on the injected volume. We find that $l_{f} / d$ is proportional to the ratio $\beta / \alpha$ (Fig. 10b) which makes it possible to predict, from the lateral and upward extent, the penetration length of the gas below the injection point - not easily accessible in the field. A linear fit gives the empirical relationship $l_{f} / d \simeq 1.3 \beta / \alpha$. For $\chi>\chi_{c}, l_{f} / d$ depends on the injected volume. This dependence is reported in figure 10c, which displays the variations of $\left(l_{f} / d\right)^{2}$ as a function of $N$. For large $\chi$ (typically, above $10 \chi_{c}$ ) we find $l_{f} / d \propto \sqrt{N}$, as expected for the invasion in an isotropic medium). As $\chi$ decreases, the symetry is broken in the system and we observe a departure from the linear tendency displayed in Figure 10c (black dots). Note finally that when $\chi$ becomes very small (high effective gravity in the system for example), $l_{f}$ tends towards a small value of the order of a few grain size, which does not increase significantly when $N$ increases.

\section{Conclusions}

This work presented a brief review on the experimental and numerical study of air invasion in an immersed granular medium. We reported additional experimental results concerning the morphology and the transient growth of the fluidized zone. It was shown that the shape is independent of the air injection flow-rate over a wide range of values, and only slightly depends on the tilt angle (effective gravity in the system). Above a critical value of this angle $\left(\alpha>60^{\circ}\right)$, the air opens a stable channel which ejects the grains from the granular layer, and a fluidized zone cannot be defined anymore. In the transient regime, we pointed out a logarithmic growth of the fluidized zone borders, in both the horizontal and vertical directions. Air rises at the center, while two convection rolls continuously rearrange the grains in the central region. A transition zone between the always-moving core (very loose packing) and the still outer region (compact) displays discrete sliding events (stick-slip zone, or SSZ). The SSZ width decreases with time, until it disappears in the stationary regime, when the fluidized zone has reached its final geometry.

We also reported a complementary numerical study of the geometry and growth dynamics of the region invaded by the gas injected in the bulk. The numerical model considers a rigid porous medium, and does not allow any grain motion. The analog of the fluidized zone corresponds to the region explored by the successive air paths through the system. This approach, although simplistic, makes it possible to extract the relevant parameters to describe the morphology of the region explored by the air. Contrary to our previous study, the gas is allowed to propagate downwards. The study provides important pieces of information on the depth reached by the injected gas. Depending on a single parameter $\chi$ which compares the gravity to the 
capillaty effects, the gas mainly propagates upwards or invades the material almost isotropically. It is found that the downward distance reached by the gas is given by the ratio of two parameters $\alpha$ and $\beta$ which describe the dynamics of the invasion pattern. In field situations, these parameters as well as the dimensionless number $\chi$ can be measured independently, and therefore provide a precise estimate of the depth reached by the gas. The proposed scaling is of practical importance as it makes possible to forecast, for instance, the potential region altered by the rupture of underground gas reservoirs.

\section{References}

1. Mörz, T., Karlik, E.A, Kreiter, S., Kopf, A.: An experimental setup for fluid venting in unconsolidated sediments: New insights to fluid mechanics and structures, Sediment. Geol. 196, 251-267 (2007).

2. Naudts, L., Greinert, J., Artemov, Y., Beaubien, S.E., Borowski, C., De Batist, M.: Anomalous sea-floor backscatter patterns in methane venting areas, Dnepr paleo-delta, NW Black Sea, Mar. Geol. 251, 253-267 (2008).

3. Semer, R., Adams, J.A., Reddy, K.R.: An experimental investigation of air flow patterns in saturated soils during air sparging: Geotech. Geol. Eng. 16, 59-75 (1998).

4. Nermoen, A., Galland, O., Jettestuen, E., Fristad, K., Podladchikov, Y., Svensen, H., Malthe-Sørenssen, A.: Experimental and analytic modeling of piercement structures, J. Geophys. Res. 115, B10202 (2010).

5. Walters, A.L., Phillips, J., Brown, R.J., Field, M., Gernon, T., Stripp G., Sparks R.S.J.: The role of fluidisation in the formation of volcaniclastic kimberlite: Grain size observations and experimental investigation, J. Volcanol. Geotherm. Res. 155, 119-137 (2006).

6. Planke, S., Svensen, H., Hovland, M., Banks, D.A., Jamtveit, B.: Mud and fluid migration in active mud volcanoes in Azerbaijan, Geo. Mar. Lett. 23, 258-268 (2003).

7. Svensen, H., Jamtveit, B., Planke, S., Chevallier, L.: Structure and evolution of hydrothermal vent complexes in the Karoo basin, South Africa, J. Geol. Soc. 163, 671-682 (2006).

8. Hovland, M., Talbot, M., Qvale, H., Olaussen, S., Aasberg, L.: Methane-related carbonate cements in pockmarks of the North Sea, J. Sediment. Petrol. 88, 881-892 (1987).

9. Wilhelm, T., Wilmański, K.: On the onset of flow instabilities in granular media due to porosity inhomogeneities, Int. J. Multiphase Flow 28, 1929-1944 (2002).

10. Rigord, P., Guarino, A., Vidal, V., Géminard, J.-C.: Localized instability of a granular layer submitted to an ascending liquid flow, Gran. Matt. 7, 191-197 (2005).

11. Zoueshtiagh, F., Merlen, A.: Effects of a vertically flowing water jet underneath a granular bed, Phys. Rev. E 75, 056313 (2007).

12. Eden, M.: A two-dimensional growth process, 4th Berkeley Symposium, Univ. California Press, 223-239 (1961).

13. Vold, M.J.: Computer simulation of floc formation in a colloidal suspension, J. Colloid Sci. 18, 684-695 (1963).

14. Sutherland, D.N.: Comment on Vold's simulation of floc formation, J. Colloid Interf. Sci. 22, 300-302 (1966).

15. Witten Jr, T.A., Sander L.M.: Diffusion-Limited Aggregation, a kinetic critical phenomenon, Phys. Rev. Lett. 47, 1400-1403 (1981).
16. Meakin, P.: The Vold-Sutherland and Eden models of cluster formation, J. Colloid Interf. Sci. 96, 415-424 (1983).

17. Martín, H., Vannimenus, J., Nadal, J.P.: From invasion to Eden growth: A family of models for cluster growth in a random environment, Phys. Rev. A 30, 3205-3213 (1984).

18. Chaouche, M., Rakotomalala, N., Salin, D., Xu, B., Yortsos, Y.C.: Invasion percolation in a hydrostatic or permeability gradient: Experiments and simulations, Phys. Rev. E 49, 4133-4139 (1994).

19. Birovljev, A., Furuberg, L., Feder, J., Jøssang, T., Måløy, K.J., Aharony, A.: Gravity invasion percolation in two dimensions: Experiments and simulation, Phys. Rev. Lett. 67, 584-587 (1991).

20. Meakin, P., Feder, J., Frette, V., Jøssang, T.: Invasion percolation in a destabilizing gradient, Phys. Rev. A 46, 3357-3368 (1992).

21. Bo, Z., Loggia, D., Xiaorong, L., Vasseur, G., Ping, H.: Numerical studies of gravity destabilized percolation in 2D porous media, Eur. Phys. J. B. 50, 631-637 (2006).

22. Chevalier, C., Lindner, A., Leroux, M., Clément, E.: Morphodynamics during air injection into a confined granular suspension, J. Non-Newton. Fluid Mech. 158, 63-72 (2009).

23. Sandnes, B., Flekkøy, E.G., Knudsen, H.A., Måløy, K.J., See, H.: Patterns and flow in frictional fluid dynamics, Nature Commun. 2, 288-296 (2011).

24. Varas, G., Vidal, V., Géminard, J.-C.: Venting dynamics of an immersed granular layer, Phys. Rev. E 83, 011302 (2011).

25. Varas, G., Vidal, V., Géminard, J.-C.: Morphology of air invasion in an immersed granular layer, Phys. Rev. E 83, 061302 (2011).

26. Lake, W.L.: Enhanced Oil Recovery, Prentice Hall (1989). 27. Eccles, J.K., Pratson, L., Newell, R.G., Jackson, R.B.: Physical and economic potential of geological $\mathrm{CO}_{2}$ storage in saline aquifers, Environ. Sci. Technol. 43, 1962-1969 (2009).

28. Romanov, V.N., Ackman, T.E., Soong, Y., Kleinman, R.L.: $\mathrm{CO}_{2}$ storage in shallow underground and surface coal mines: challenges and opportunities, Environ. Sci. Technol. 43, 561-564 (2009).

29. Kang, Q., Tsimpanogiannis, I.N., Zhang, D., Lichtner, P.C.: Numerical modeling of pore-scale phenomena during $\mathrm{CO}_{2}$ sequestration in oceanic sediments, Fuel Process. Technol. 86, 1647-1665 (2005).

30. Svensen, H., Planke, S., Malthe-Sørenssen, A., Jamtveit, B., Myklebust, R., Eldem, T.R., Rey, S.: Release of methane from a volcanic basin as a mechanism for initial Eocene global warming, Nature 429, 542-545 (2004).

31. Kong, X.-Z., Kinzelbach, W., Stauffer, F.: Morphodynamics during air injection into water-saturated movable spherical granulates, Chem. Eng. Sci. 65, 4652-46660 (2010).

32. Melo, F., Vivanco, F., Fuentes, C., Apablaza, V.: On drawbody shapes: From BergmarkRoos to kinematic models, Int. J. Rock Mech. Mining Sci. 44, 77-86 (2007). 